\title{
Erratum to: Proteomics and diabetic nephropathy: what have we learned from a decade of clinical proteomics studies?
}

Massimo Papale • Salvatore Di Paolo •

Grazia Vocino - Maria Teresa Rocchetti •

Loreto Gesualdo

Published online: 16 July 2014

(C) Italian Society of Nephrology 2014

Erratum to: J Nephrol

DOI 10.1007/s40620-014-0044-5

A faulty version of Fig. 1 was submitted for publication.

The corrected figure is reproduced here.

The online version of the original article can be found under doi:10.1007/s40620-014-0044-5.

M. Papale

Core Facility of Proteomics and Mass Spectrometry,

Department of Surgery and Medical Sciences,

University of Foggia, Foggia, Italy

S. Di Paolo

Nephrology Unit, “Dimiccoli” Hospital, Barletta, Italy

G. Vocino $\cdot$ M. T. Rocchetti $\cdot$ L. Gesualdo $(\bowtie)$

Nephrology, Dialysis and Transplantation Unit,

Department of Emergency and Organ Transplantation (D.E.T.O),

University of Bari "Aldo Moro", Bari, Italy

e-mail: segrnefr@gmail.com 


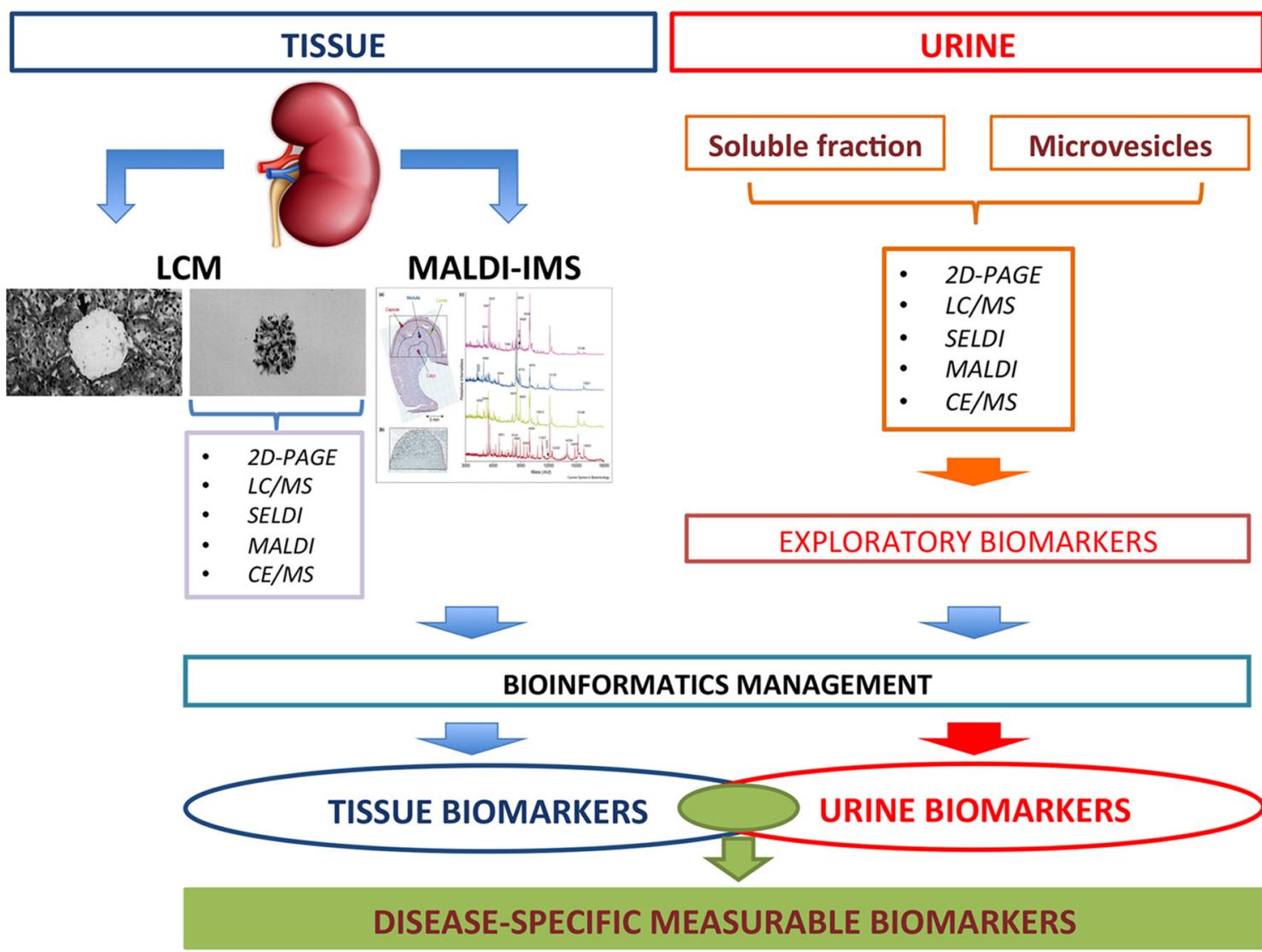

Fig. 1 Workflow of the proteomic analysis applied to tissue and urine samples. Left panel Kidney sub-compartments (i.e. glomeruli, tubuli, etc.) can be isolated from whole biopsy specimens through laser capture microdissection (LCM) prior to performing proteomic analysis. Alternatively, frozen or formalin fixed and paraffin embedded (FFPE) tissues can be directly analysed by matrix-assisted laser desorption/ionization imaging mass spectrometry (MALDI-IMS). Right panel Ultracentrifugation of whole urine samples is used to separate soluble and vesicular fractions, then proteomic analysis may allow the identification of compartment-specific biomarkers. The bioinformatic management of urine and tissue putative biomarkers is then applied to each dataset in order to select functionally correlated disease biomarkers [72]. Comparative analysis may finally allow to identify tissue-derived biomarkers also detectable in the urine samples. LCM glomeruli and MALDI-IMS figures are reproduced with permission from Kohda et al. [73] and Chaurand et al. [74], respectively 